\title{
Häufigkeit, Entstehung und Klassifikation der distalen Radiusfraktur
}

\author{
Sebastian Schmidt, Tim Heinz, Andreas Dávid
}

\section{Zusammenfassung}

In der Literatur wird eine Vielfalt unterschiedlicher Klassifikationen distaler Radiusfrakturen beschrieben, mit deren Hilfe man versucht, ein optimales Behandlungskonzept $\mathrm{zu}$ finden und eine möglichst genaue Abschätzung des Behandlungsergebnisses zuzulassen. Es steht außer Frage, dass es das Ziel sein sollte, bei jeder Therapieform frischer Knochenverletzungen, eine möglichst gute Gelenkfunktion zu erhalten, eine Ausheilung in Fehlstellung und eine posttraumatische Arthrose zu verhindern. Ein gutes System zur Einteilung distaler Radiusfrakturen sollte deswegen beschreibend und gleichzeitig auch voraussagend sein, wobei eine Auswahl der geeigneten Behandlungsformen wie z.B. geschlossene oder offene Reposition, transkutane oder offene Osteosyntheseverfahren und die Anwendung von autologen Knochenmarktransplantationen miteingehen muss. Die Arbeit soll einen Überblick über die Frakturklassifikationen distaler Radiusfrakturen geben, die in der gegenwärtigen Literatur zu finden sind und den Stellenwert einer Fraktureinteilung am Gesamtbehandlungskonzept am Beispiel der distalen Radiusfraktur herausstellen. Am weitesten verbreitet sind die Klassifikationen der AO und die Klassifikation nach Frykman.

\section{Häufigkeit}

Die distale Radiusfraktur ist mit 17\% die häufigste Knochenverletzung aller Frakturen $[1,2]$. Sie steht bei Verletzungen der oberen Extremität mit $14 \%$ und bezogen auf Unterarmfrakturen sogar mit 75\% an erster Stelle [3].

Die Geschlechts- und Altersverteilung zeigt einen biphasischen Verlauf. Im jüngeren Lebensalter handelt es sich vorwiegend um männliche Patienten, mit Arbeits- und Sportunfällen. Im höheren Alter dominiert das weibliche Geschlecht, wobei hier die postmenopausale Osteoporose mit verminderter Knochendichte ein wesentlicher Faktor der Frakturgenese ist $[4,5]$.

OP-JOURNAL 2003; 19: 10-15

(C) Georg Thieme Verlag Stuttgart - New York

\section{Entstehung}

$90 \%$ aller distaler Radiusfrakturen sind Extensionsfrakturen. Sie entstehen durch Sturz auf das überstreckte Handgelenk, wobei unter axialer, indirekter Stauchung, die durch den Bruch entstehenden Knochenfragmente unterschiedlich gegeneinander verschoben werden.

Die Bruchform ist abhängig von Größe und Richtung der einwirkenden Kraft. Anatomisch gesehen ist der distale Radius das proximale Ende des Radiokarpalgelenks. Es besteht aus zwei konkaven radialen Gelenkfacetten und dem Diskus articularis, die mit dem Os skaphoideum und dem Os lunatum artikulieren. Über ein volares und dorsales Ligament wird der Karpus am distalen Radius stabilisiert und befestigt. Diese Gelenkstrukturen spielen eine wichtige funktionelle Rolle bei Rotationsbewegungen der Hand und des Radius gegenüber der fixierten Ulna [6].
Die Lokalisation der Fraktur wird zudem wesentlich vom Winkel der Krafteinwirkung zum Handgelenk bestimmt. Eine distale Radiusextensionsfraktur entsteht bei einem Winkel zwischen Unterarm und Handwurzel von $40^{\circ}$ und $90^{\circ}$. Darunter kommt es zu einer Unterarmfraktur, und darüber zu Luxationen und Frakturen im Handwurzelbereich. Typischerweise befindet sich die Frakturstelle etwa 1,5 bis $2 \mathrm{~cm}$ proximal der Radiusgelenkfläche. Durch dorsale Dislokation entsteht die typische Gabelstellung des Handgelenks. Häufig ist bei einer Fraktur des Proc. styloideus ulnae der Discus articularis mitverletzt. Die intraartikulären Frakturen sind zumeist Mehrfragmentfrakturen. Bei einem Sturz auf den flektierten Handrücken kommt es dagegen zu einer volaren Absprengung.

\section{Klinik}

Wesentliche Symptome der distalen Radiusfraktur sind die schmerzhafte Schwellung, eine Verbreiterung oder Fehlstellung des Handgelenks mit eingeschränkter Beweglichkeit. Die Patienten klagen oft über ein Instabilitätsgefühl. Weiterhin können Sensibilitätsstörungen der Hand oder der Finger auftreten.

Kann ein Sturz mit Radiusfraktur nicht auf einen Unfall zurückgeführt werden, muss immer nach anderen Ursachen gesucht werden.

So kann es sich um einen Sturz bei Herzrhythmusstörungen oder zerebraler Durchblutungsstörung handeln. Möglicherweise ist ein Bagatelltrauma bei osteoporotischer Begleiterkrankung die Ursache für die Verletzung. Auch Vorerkrankungen (neurologische Erkrankungen, Karpaltunnelsyndrom, Ulnaris-Rinnensyndrom) und vorausgegangene Verletzungen (Handwurzelluxationen, Scaphoidfraktur und karpale Instabilität) müssen erfasst werden. 
Bei der klinischen Untersuchung lassen sich Prellmarken, Hämatome, Druckschmerz und Bewegungsschmerz feststellen. Es ist notwendig die aktive Funktion der Fingergelenke, die Daumenstrecker-Funktion zu überprüfen, sowie evtl. neurologische Ausfälle und Durchblutungsstörungen zu erkennen.

Bei schweren Verletzungen, insbesondere bei erst- bis drittgradig offenen Frakturen, muss immer an Weichteilschäden mit sich entwickelndem Kompartmentsyndrom gedacht werden. Eine Schädigung des N. medianus ist auch möglich.

Die Röntgenuntersuchung des Handgelenks in zwei Ebenen gibt Klarheit darüber, ob und welche Form einer Fraktur vorliegt, und wieweit das Handgelenk in Mitleidenschaft gezogen worden ist. Die sich aus klinischer und radiologischer Diagnostik ergebende Frakturart und ihre Begleitverletzungen bestimmen das weitere konservative oder operative Vorgehen. In der Literatur wird eine Vielzahl von Einteilungsmöglichkeiten für distale Radiusfrakturen beschrieben.

\section{Klassifikation}

Die klassische Einteilung der distalen Radiusextensionsfrakturen erfolgt nach Pouteau 1783 und Colles 1814 [7] und die der Radiusflexionsfrakturen nach Smith 1847.

Die Frakturen nach Colles werden in vier Klassen unterteilt [8]. Man spricht auch von der „Fractura loco typico sive classico“ (Abb.1). Nachteil ist, dass bei dieser Einteilung nur Extensionsfrakturen berücksichtigt werden und die Bezeichnung
Tab. 1 Frykman-Klassifikation

\begin{tabular}{|c|c|}
\hline \multicolumn{2}{|c|}{ Quere extraartikuläre Frakturen } \\
\hline Frykman I & $\begin{array}{l}\text { Extensionsfraktur nach Pouteau-Colles und Flexionsfraktur nach Goyrand- } \\
\text { Smith, ohne Gelenkbeteiligung }\end{array}$ \\
\hline Frykman II & wie Frykman I, zusätzlich Fraktur des Proc. styloideus ulnae \\
\hline \multicolumn{2}{|c|}{ Intraartikuläre Frakturen mit Beteiligung des Radiokarpalgelenks } \\
\hline Frykman III & Schrägfraktur des Radius (Fraktur des Proc. styloideus radii) \\
\hline Frykman IV & wie Frykman III, zusätzlich Fraktur des Proc. styloideus ulnae \\
\hline \multicolumn{2}{|c|}{ Beteiligung des Radioulnargelenkes } \\
\hline Frykman V & Querfraktur mit Gelenkbeteiligung \\
\hline Frykman VI & wie Frykman V, zusätzlich Fraktur des Proc. styloideus ulnae \\
\hline \multicolumn{2}{|c|}{ Kombination der Gelenkbeteiligung beider Gelenke } \\
\hline Frykman VII & Kombination aus Frykman III und V \\
\hline Frykman VIII & wie Frykman VII, zusätzlich Fraktur des Proc. styloideus ulnae \\
\hline
\end{tabular}

nicht auf die unterschiedlichen Formen der distalen Radiusfraktur eingeht, die ja, je nach Art, unterschiedliche Behandlungsmethoden verlangt.

Colles I: extraartikulär, nicht disloziert Colles II: extraartikulär, disloziert Colles III: intraartikulär, nicht disloziert Colles IV: intraartikulär, disloziert

Eine weit verbreitete Einteilung der Extensionsfrakturen ist die nach Frykman [9] (Abb.2). Unterteilt wird in extraund intraartikuläre Frakturen mit und ohne Beteiligung des Proc. styloideus ulnae. Diese Einteilung vernachlässigt eine Differenzierung der Flexionsfrakturen, da sie sich am posterioren-anterioren Strahlengang orientiert. Außerdem geht sie nicht auf Ausdehnung und Richtung einer etwaigen Dislokation von Frakturfragmenten ein. Ebenso werden die dorsale Trümmerzone und die Radiusverkürzung

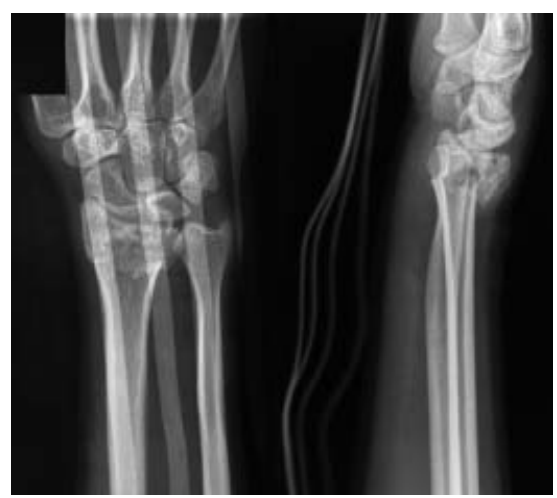

Abb. 1 Typisches Bild einer distalen Radiusfraktur. (links) Im a.p. Strahlengang lässt sich die Fraktur im Bereich der Metaphyse erkennen. Es imponiert die Radialabweichung der Hand mit Prominenz des Proc. Styloideus ulnae. (rechts) Der seitliche Strahlengang zeigt die typische Bajonett- oder FourchetteStellung mit Abkippung des distalen Radius nach dorsal.

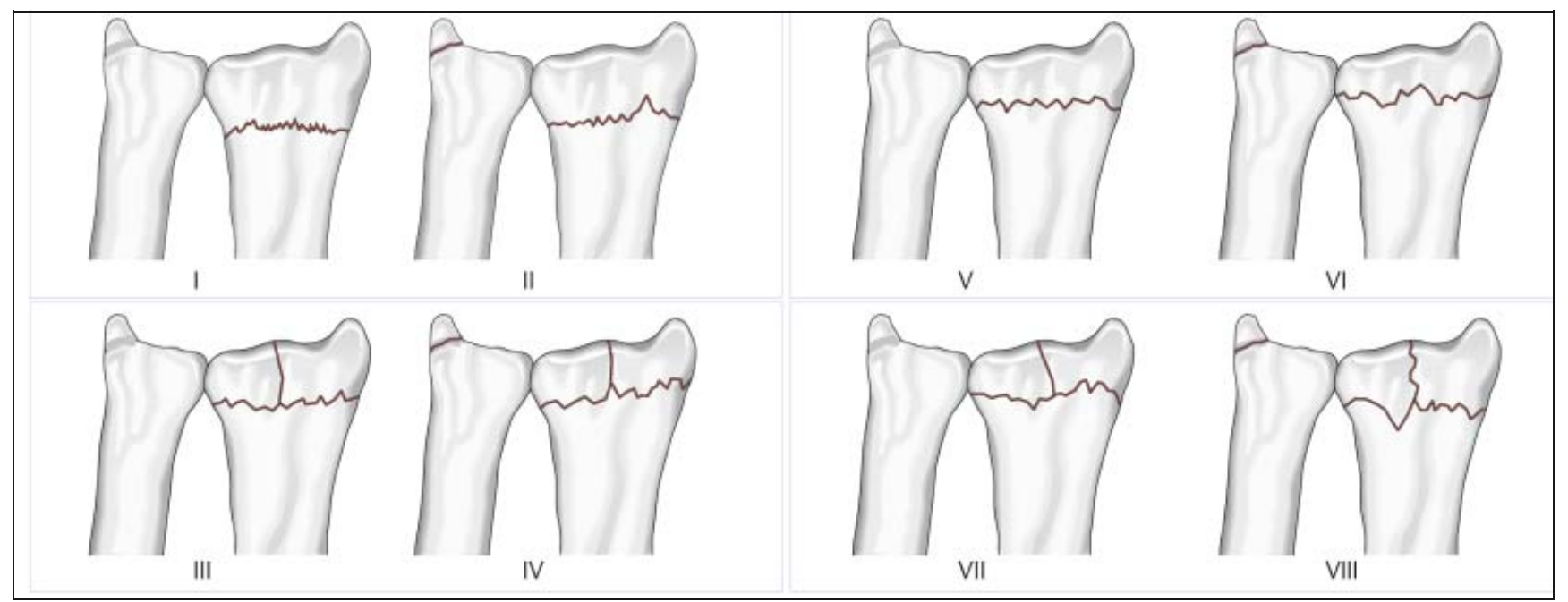

Abb. 2 Einteilung nach Frykman (1967). Berücksichtigt wird bei dieser Einteilung die ossäre Verletzung des Radioulnargelenks. Je höher die Typeneinteilung desto schwerwiegender ist die Knochenverletzung. 


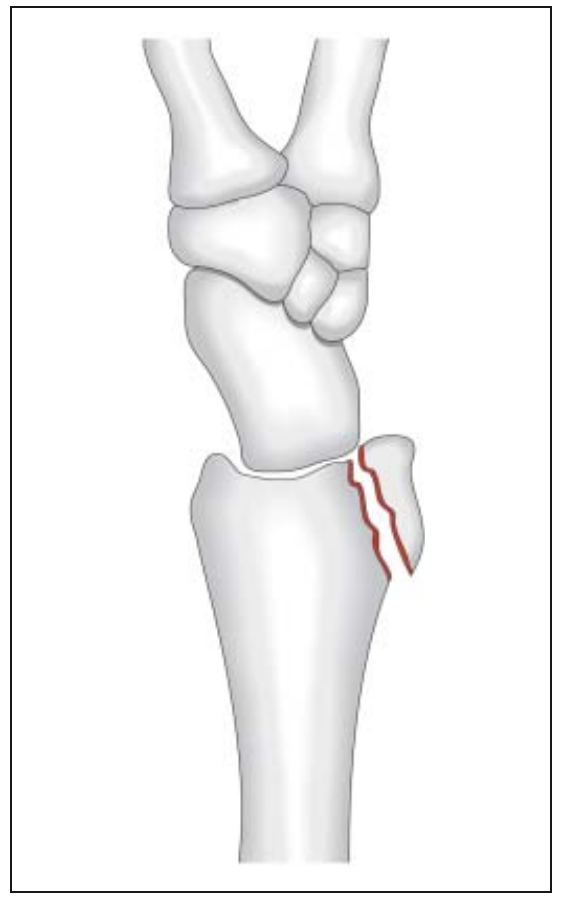

Abb. 3 Dargestellt wird eine Sonderform der Radiusextensionsfraktur. Kantenabsprengung bzw. Ausbruch eines Knochenfragments an der dorsalen Kante der Radiusgelenkfläche (Barton-Fraktur).

nicht berücksichtigt. Die Frykman-Klassifikation lässt keine Rückschlüsse auf eine Behandlungsindikation zu. Eine Aussage über die Stabilität der Fraktur ist nur schwer möglich $[10,11]$.

Die Einteilung nach Kuner stellt ebenfalls eine Vereinfachung der Systematisierung der Extensionsfrakturen dar. Sie basiert auf der Klassifikation von Frykman, ohne die Abrissfrakturen des Proc. styloideus ulnae zu berücksichtigen [12].

Frakturklassifikation nach Kuner Typ A: extra-artikuläre Fraktur Typ B: radiokarpale Gelenkfraktur Typ C: radioulnare Gelenkfraktur Typ D: radiokarpale und radioulnare Gelenkfraktur

Die Barton-Fraktur (Abb.3) gehört zu den Radiusextensionsfrakturen und beschreibt intraartikuläre dorsale 2-Fragment-Kantenabbrüche mit oder ohne Subluxation des Karpus. Ihr Anteil an den distalen Radiusfrakturen liegt zwischen $1,2 \%$ und $2,3 \%$ [13].

Bei der Radiusflexionsfraktur lässt sich ein volares Kantenfragment finden. Smith beschreibt hier die Lage des dislozierten volaren Kantenfragments in Bezug zur radialen Gelenkfläche. Weiterhin

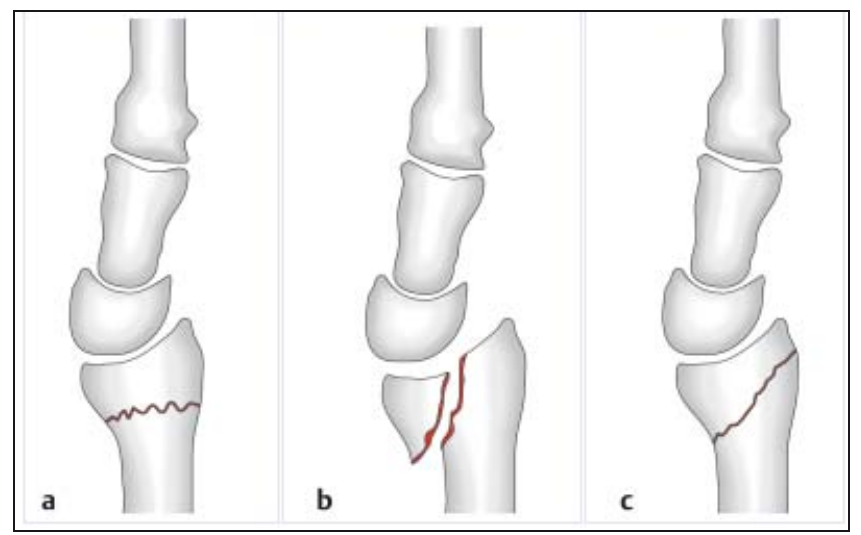

Abb. 4 Klassifikation der Smith-Frakturen: Die Abbildung zeigt Flexionsfrakturen des distalen Radius mit volarer Abkippung oder der Tendenz dazu. (a) Extra-artikuläre Smith-Fraktur mit horizontalem Bruchspalt (Typ Thomas 1). (b) Intra-artikuläre Smith-Fraktur (Typ Thomas 2 bzw. Reversed Barton). (c) Extra-artikuläre Smith-Fraktur mit schräg verlaufendem Bruchspalt (Typ Thomas 3). Modifiziert n. Oliviera J. C.: Barton's fracture. J Bone Joint Surgery 1973; 55A: 586-594).

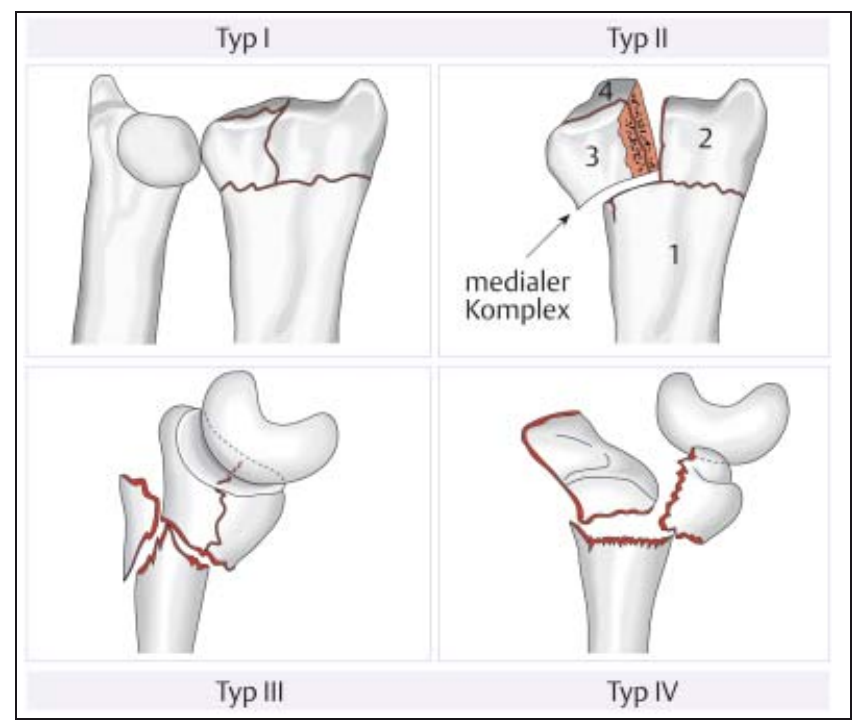

Abb.5 Die frakturierte Gelenkfläche wird nach Melone in 4 Schlüsselfragmente unterteilt. (1) Radiusschaft, (2) radiales Styloidfragment, (3) dorso-ulnares Fragment, (4) palmar-ulnares Fragment. Bei Dislokation des medialen Komplexes ist gleichzeitig das Radiokarpal- und Radioulnargelenk mitbeteiligt. Bei großer Krafteinwirkung wird der mediale Komplex weit auseinandergedrängt, so dass es zu einer schweren Zerstörung des gesamten Gelenkverbundes kommt (Modifiziert n. Melone C.P. Jr.: Articular fractures of the distal radius. Orthop Clin North Am 1984; 15: 217-236).

existiert eine Einteilung der Smith-Frakturen nach Thomas, wobei hier die Frakturlinie von entscheidender Bedeutung ist (Abb.4). Thomas Typ II wird auch als Reversed-Barton-Fraktur bezeichnet, bei der es nach volarem Kantenabriss zur vorderen Subluxation des Karpus kommt.

Einteilung nach Thomas

Typ I: extra-artikuläre Fraktur mit metaphysär verlaufendem Bruchspalt

Typ II: volare Luxationsfraktur mit Beteiligung der distalen Radiusgelenkfläche
Typ III: extraartikuläre Schrägfraktur des distalen Radius

Die Einteilung intraartikulärer 4-Fragment-Frakturen nach Melone [14], basiert auf der Analyse radiokarpaler und radioulnarer Schlüsselfragmente (1. Radiusschaft, 2. radiales Styloidfragment, 3. dorso-ulnares Fragment, 4. palmar-ulnares Fragment). Das dritte und vierte Fragment bildet zusammen mit den ligamentären Verbindungen zum Proc. styloideus ulnae und der proximalen Reihe der Handwurzelknochen den sogenannten medialen Komplex (Abb.5). Durch- 
schnittlich $50 \%$ der distalen Radiusfrakturen sind Typ-II-Frakturen mit Einstauchung des dorso-ulnaren Fragments durch das OS lunatum.

\section{Frakturklassifikation nach Melone}

Typ I: stabil, wenig eingestaucht

Typ II: instabil, med. Komplex im Verbund disloziert

Typ III: instabil, med. Komplex im Verbund disloziert, zusätzliches volares Schaftfragment

Typ IV: instabil, weite Dislokation des dorsalen (3) und volaren (4) Schlüsselfragments

Die einzige Klassifikation neben der AOKlassifikation, die auch Mehrfragmentbrüche kategorisiert und gleichzeitig Dislokationen mitberücksichtigt, ist die Einteilung von Older [15]. Die Einteilung erfolgt nach dem Grad der dorsalen Abkippung, Dislokation, Radiusverkürzung* und dorsaler metaphysärer Einstauchung.

Klassifikation nach Older

Typ1: dorsale Abkippung $5^{\circ}$, Radiusverkürzung $7 \mathrm{~mm}$

Typ2: dorsale Abkippung $5^{\circ}$, Radiusverkürzung $1-7 \mathrm{~mm}$

Typ3: dorsale Abkippung $5^{\circ}$, Radiusverkürzung $4 \mathrm{~mm}+$ wenig eingestaucht

Typ4: dorsale Abkippung 5 Radiusverkürzung neg. + deutlich eingestaucht + intra-artikuläre Beteiligung

${ }^{*}$ Radiusverkürzung $=$ distal bis ulna

Eine Einteilung für intra-artikuläre Frakturen konnte durch McMurtry und Jupiter definiert werden [16]. Hierbei hängt die Einteilung von der Anzahl bestimmter Frakturelemente ab. Je höher die Typenzahl, desto komplexer die Fraktur.

Fernandez [17] bezog bei seiner Klassifikation den Unfallmechanismus mit ein, so dass der Zusammenhang von Pathomechanismus und Morphologie gut zum Ausdruck kommt (Abb.6).

\section{AO-Klassifikation}

Eine umfassendere und auf den ersten Eindruck kompliziert wirkende Fraktureinteilung, ist die AO-Klassifikation (Arbeitsgemeinschaft für Osteosynthese)

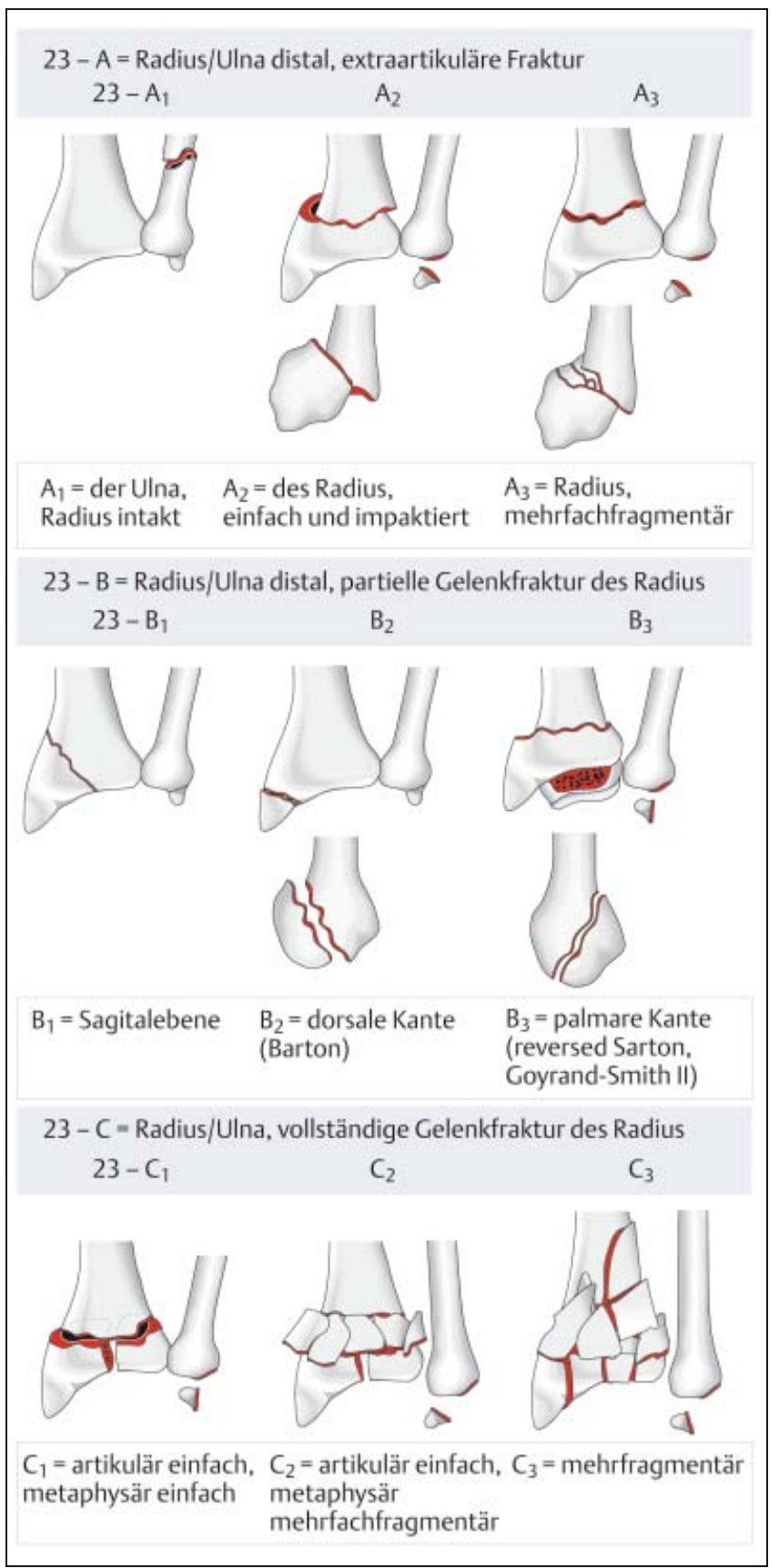

Abb. 6 Klassifikation der distalen Radiusfraktur nach Fernandez. Typ 1: Biegungsfraktur Die Metaphyse kippt in Belastungsrichtung, Typ 2: Kompressionsfraktur Gelenkflächenbeteiligung mit Einstauchung des subchondralen Knochens, Typ 3: Abscherfraktur - Gelenkflächenbeteiligung (Bartonfraktur), Typ 4: Abrissfraktur - ligamentäre Beteiligung (Fraktur von Proc. styloideus ulnae et radii), Typ 5: Kombination aus Typ 1-4 und Hochenergietraumen.

nach Müller [18] (Abb.7). Sie besitzt den Vorteil einer direkten Umsetzbarkeit in ein therapeutisches Vorgehen. Jeder lange Röhrenknochen (oder Röhrenknochenpaar) wird mit einer Ziffer versehen und jeder Knochen in drei Segmente (proximal, diaphysär und distal) unterteilt, wobei das Zentrum der Fraktur für die Zuordnung entscheidend ist. Für den distalen Unterarm ergibt sich dadurch die Knochensegmentzahl 23. Die Frakturen werden weiterhin in die Typen A, B und C aufgeteilt, die das Ausmaß der Gelenkflächenbeteiligung bestimmen. Jeder Typ besteht aus drei Gruppen, mit je drei Untergruppen. So kommt man insgesamt auf 27 Frakturtypen des distalen Unterarms. Die Gruppe A1 bildet die isolierten Ulnafrakturen. Die Gruppe A2 (Abb.8a) teilt einfache extra-artikuläre Frakturen ohne Trümmerzone, und die Gruppe A3 die mit Trümmerzone ein. Zur Gruppe A2 gehören auch die einfachen Frakturen nach Colles und Smith. Auf einen Abriss des Proc. styloideus ulnae geht die AO-Klassifikation erst in nachfolgenden Subgruppen ein.

Die Gruppe B1 teilt partiell artikuläre Frakturen mit sagittaler Frakturlinie, die 


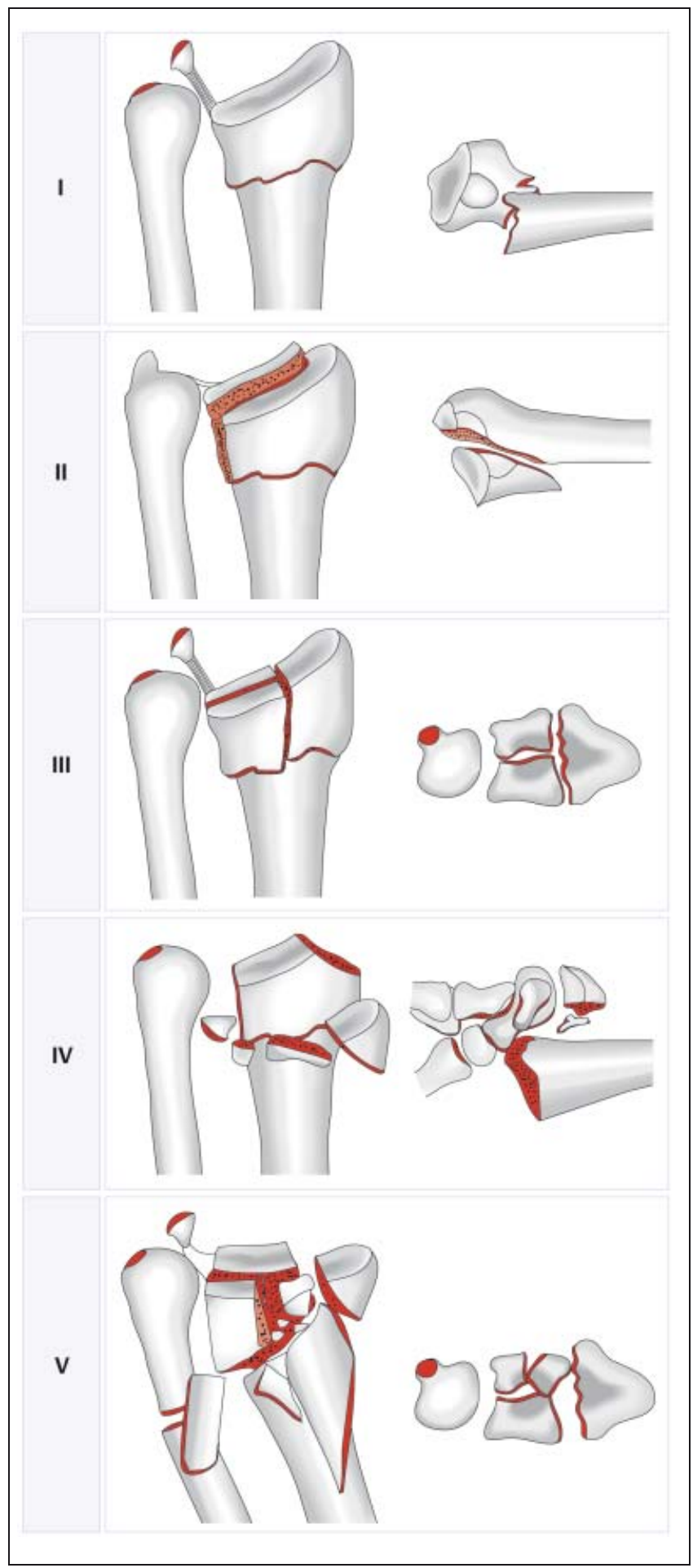

Abb. 7 AO-Klassifikation nach Müller. 23-A. Extraartikuläre Frakturen, 23-B. Partielle Gelenkfrakturen, 23-C. Vollständig artikuläre Frakturen.
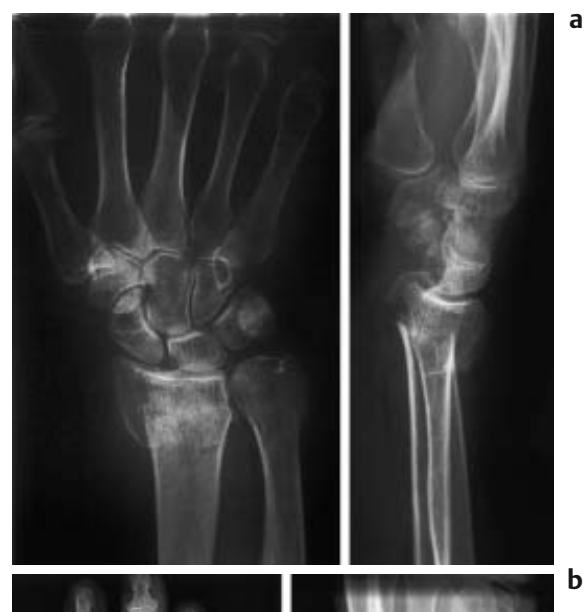

b
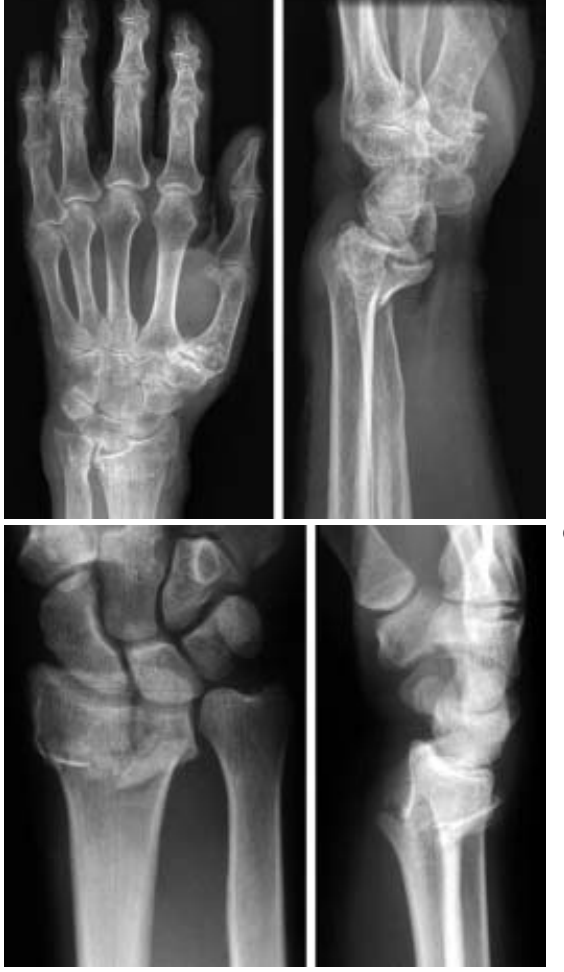

Abb.8 (a) Extraartikuläre distale Radiusfraktur Typ A2 mit dorsaler Abkippung. (b) Partielle Gelenkfraktur mit volarem Kantenabriss Typ B3 (c) Einfache Gelenkfraktur, metaphysär mehrfragmentär Typ C2.

mit subkapitaler Ulnafraktur und Ulnafraktur proximal des Halses.

Die AO-Klassifikation erlaubt die Zuordnung einer Fraktureinteilung für jeden Frakturtyp, anhand von Röntgenaufnahmen in nur zwei Ebenen. Darüber hinaus lässt sich eine Differenzialindikation für die weitere Therapie stellen, die in den nachfolgenden Artikeln differenziert dargestellt werden. Die Frakturen der Gruppen A2 und A3 bleiben im Wesentlichen eine Domäne der konservativen Therapie, evtl. ergänzt durch die Kirschner-Draht-
Die Untergruppen können bei zusätzlichen Verletzungen der Ulna, jeweils noch in sechs Subgruppen eingeteilt werden. Hierbei werden berücksichtigt: Abrissfraktur des Proc. styloideus ulnae, einfache subkapitale Ulnafraktur, Mehrfragmentfraktur des Ulna-Halses, Köpfchenfraktur der Ulna, Ulnaköpfchenfraktur Frakturen mit einfacher artikulärer und metaphysärer Beteiligung. Die Gruppe C2 (Abb.8c) teilt die artikulär einfachen und metaphysär mehrfragmentären Frakturen ein. Die Gruppe C3 beinhaltet arti- kuläre wie metaphysäre Mehrfragment- 
spickung. Für die Frakturen des Typs B kommen vornehmlich Abstützplatten und für die Fraktur vom Typ C der Fixateur externe in Betracht.

Auch die Prognose einer Fraktur kann, eine adäquate Therapie vorausgesetzt, aus der Klassifikation hergeleitet werden. Wittner bemängelt bei der AO-Klassifikation lediglich, dass die A-Gruppen nicht auf die ligamentären Begleitverletzungen und die C-Gruppen nicht ausreichend auf die Dislokation der Fragmente eingehen [11].

\section{Schlussfolgerung}

Für die Entscheidung eines angemessenen Therapieverfahrens ist es sicherlich wichtig, vorab zu klären, in welchem Ausmaß das Radiokarpalgelenk geschädigt wurde, und wie die Stabilität nach Reposition einzuschätzen ist. Im deutschsprachigen Raum hat sich deswegen die Einteilung der handgelenksnahen Unterarmfrakturen anhand der AO-Klassifikation durchgesetzt. Aus der Einteilung sollte sich dann die therapeutische Konsequenz herleiten. Nach Wittner sollte eine Klassifikation umfassend, eindeutig, anwendbar und überschaubar sein [11]. Auch sollte sie eine prognostische Aussage zulassen.

Weil wahrscheinlich keine Fraktureinteilung allein diese Bedingungen erfüllen kann, sollte idealerweise zur Behandlung der distalen Radiusfraktur die Differenzierung zwischen intra/extra-artikulären Frakturen, die Beurteilung von Dislokationen, die Ausdehnung von Trümmerzonen und das Erfassen ligamentärer Begeleitverletzungen angestrebt werden.

\section{Literatur}

${ }^{1}$ Owen RA, Melton LJ, III, Johnson KA et al.. Incidence of Colles' fracture in a North American community. Am J Public Health 1982; 72: 605-607

2 Palmer AK. Fractures of the distal radius. In: Green D. Operative Hand Surgery. ChurchillLivingstone, New York.1988, S. 991-1026

${ }^{3}$ Alffram PA, Bauer GCH. Epidemiology of fractures of the forearm: A biomechanical investigation of bone strenght. J Bone Joint Surg 1962; 44a: 105-114

${ }^{4}$ Kaps HP. Wann konservativ, wann operativ, wie akut, wie langfristig behandeln? Krankenhausarzt 1994; 67: 405-414

${ }^{5}$ Petracic B. Treatment of distal multi-fragment joint fractures of the radius. Indications, technique and results. Chirurg 1993; 64: $894-898$

${ }^{6}$ Jupiter JB. Fractures of the distal end of the radius. J Bone Joint Surg Am 1991; 73: 461 469

${ }^{7}$ Colles A. Historical paper on the fracture of the carpal extremity of the radius (1814). Injury 1970; $2: 48-50$

8 Sarmiento A, Pratt GW, Berry NC et al. Colles' fractures. Functional bracing in supination. J Bone Joint Surg Am 1975; 57: 311 - 317

${ }^{9}$ Frykman G. Fracture of the distal radius including sequelae-shoulder-hand-finger syndrome, disturbance in the distal radioulnar joint and impairment of nerve function. A clinical and experimental study. Acta Orthop Scand 1967: Suppl

${ }^{10}$ Brunner U, Habermeyer P, Schweiberer L. Fractures of the distal end of the radius. Orthopade 1989; 18: 214-224

${ }^{11}$ Wittner B, Holz U. General classification of distal radius fractures and treatment of extra-articular distal radius fractures (Type A2 and A3). Chirurg 1993; 64: 880-888
${ }^{12}$ Kuner EH, Schlosser V. Traumatologie. Georg Thieme Verlag, Stuttgart 1988

${ }^{13}$ Pattee GA, Thompson GH. Anterior and posterior marginal fracture-dislocations of the distal radius. An analysis of the results of treatment. Clin Orthop 1988: 183-195

${ }^{14}$ Isani A, Melone CP, Jr. Classification and management of intra-articular fractures of the distal radius. Hand Clin 1988; 4: 349-360

${ }^{15}$ Older TM, Stabler EV, Cassebaum WH. Colles fracture: Evaluation and selection of therapy. J Trauma 1965; 5: 459-476

${ }^{16}$ McMurtry RY, Jupiter JB. Fractures of the distal radius. In: Browner B.D., J.B.L.A.M.Jupiter, P.T.Trafton (eds.): Skeletal Trauma: Fractures, Dislocationes, Ligamentous Injuries. WB Saunders, Philadelphia.1992, S. 1063 1094

${ }^{17}$ Fernandez DL. Fractures of the distal radius: operative treatment. Instr Course Lect 1993; 42: $73-88$

${ }^{18}$ Muller ME, Nazarian S, Koch P. Classification AO des fractures 1: Les Os Longs. Springer, Berlin 1987

\section{Dr. med. Sebastian Schmidt \\ Arzt im Praktikum \\ Dr. med. Tim Heinz \\ Assistenzarzt \\ Prof. Dr. med. Andreas Dávid}

Ärztlicher Direktor

Klinik für Unfall- und Wiederherstellungschirugie

HELIOS Klinikum Wuppertal

Heusnerstr. 40

D-42283 Wuppertal 